\title{
Comment on the Recent Start of a New "IUPAC-Project"
}

\section{Reinhard Brandt ${ }^{1}$, Valery Ditlov ${ }^{2}$, Elena Firu ${ }^{3}$, Eberhard Ganssauge ${ }^{4}$, Maria Haiduc ${ }^{3}$, Reza Hashemi-Nezhad ${ }^{5}$, Alina Tania Neagu ${ }^{3}$, Wolfram Westmeier ${ }^{1,6^{*}}$}

\author{
${ }^{1}$ Kernchemie, FB Chemie, Philipps-Universität Marburg, Marburg, Germany \\ ${ }^{2}$ Institute of Theoretical and Experimental Physics, Moscow, Russia \\ ${ }^{3}$ Bucharest Institute of Space Science, Bucharest, Romania \\ ${ }^{4}$ FB Physik, Philipps-Universität Marburg, Marburg, Germany \\ ${ }^{5}$ School of Physics, The University of Sydney, Sydney, Australia \\ ${ }^{6}$ Dr. Westmeier GmbH, Ebsdorfergrund, Germany \\ Email: ^info@westmeier.com
}

How to cite this paper: Brandt, R., Ditlov, V., Firu, E., Ganssauge, E., Haiduc, M., Hashemi-Nezhad, R., Neagu, A.T. and Westmeier, W. (2018) Comment on the Recent Start of a New "IUPAC-Project". World Journal of Nuclear Science and Technology, 8, 121-127.

https://doi.org/10.4236/wjnst.2018.83010

Received: May 16, 2018

Accepted: June 26, 2018

Published: June 29, 2018

Copyright $\odot 2018$ by authors and Scientific Research Publishing Inc. This work is licensed under the Creative Commons Attribution International License (CC BY 4.0)

http://creativecommons.org/licenses/by/4.0/

\begin{abstract}
The opening of a new IUPAC-project is highly appreciated. In the year 2009, the IUPAC had published an article "Discovery of the element with atomic number 112 (IUPAC Technical Report)" [1]* which contains a section on the work of the Marinov collaboration. It appears that this section is not always in agreement with conventional standards for scientific publications. This present comment focuses on these formal questions.
\end{abstract}

\section{Keywords}

IUPAC Project 2017-014-2-200, eka-Hg, Unresolved Problems

\section{Introduction}

The International Union of Pure and Applied Chemistry (IUPAC) has recently started a new project with the identification: "Project No: 2017-014-2-200" [2]. The central aim of this project is (quote): Over twenty-five years have elapsed since criteria that are currently used to verify claims for the discovery of a new element were set down... It is proposed to set up a project for an IUPAC/IUPAP Joint Working Group (JWG) to examine and update these criteria. (End of quote)

This appears to be a timely project, as there are questions as to whether or not the standards for scientific publications have been followed by the IUPAC in ${ }^{\star}$ References relating to this paper are given as usual and as listed in the end of this paper. References in double parentheses, e.g. [[18]], are taken from the reference list in the IUPAC-paper [1]. 
their former publication [1].

Nearly all sections of that paper are well-written, in particular those sections concerning the work of Hofmann and colleagues. However, there are some problems with the standards for scientific publications when it comes to the work of the Marinov collaboration. These inconsistencies are discussed in this article.

\section{Remarks Concerning Statements about the Marinov Collaboration}

Problems dealing with the Marinov collaboration in [1] are considered step by step.

\subsection{Problem 1}

Ref. [1] (re: Marinov) quoting page 1336-1337:

"This collaboration reiterates arguments for their discovery of the element with atomic number 112 through the existence of very long-lived hyper-deformed isomeric states of actinides [actinoids] and transactinides [tranactinoids], produced from multi-GeV protons in a thick $W$ target and, in the case of eka- $\mathrm{Hg}$, of subsequent spontaneous fission, a very nonspecific indicator. Unusually high fusion cross-sections induced by secondary products are required for nuclide formation, each several orders of magnitude beyond known behavior. Results from other research groups that attempted obvious corroboration studies using multi-GeV protons incident on a $U$ target clearly indicated the production path was irreproducible as previously stressed [[1] [3] [4]]. The latter refutations have been challenged by Brandt [[18] [25]], a member of the Marinov collaboration, in which he rejects the negative results because the same exact experiment as conducted by Marinov was not followed. However, independent evidence is what "Criteria" (q.v.) demand. Cloning of methodology is an approach that could easily camouflage systematic error."

Problem 1: Reference [[18]] has nothing to do with Brandt, he is not even an author of that paper. Moreover, [1] states that Cloning of methodology... could easily camouflage systematic error. The repetition of an experiment is part of any standard procedure and it is trivial, that an experimental procedure may include a systematic error. It is a reality that Marinov and collaborators have not repeated their first experiment from 1971. In 1971 Marinov et al. published two papers in NATURE [3a] [3b] about the production of a new element with nuclear charge $Z=112$ using an original, completely new and unexplored experimental method: They bombarded consecutively two metallic tungsten rods (nuclear charge $\mathrm{Z}=74$ and atomic weight $\mathrm{A}=183.5$ ) with $24 \mathrm{GeV}$ protons at the PS-accelerator in CERN, Geneva (Switzerland). The first target (W1) was irradiated for one year with a total flux of $1 \times 10^{18}$ protons and chemical procedures started 3 months later. The second target (W2) was irradiated for 4 months with $7 \times 10^{17}$ protons. The chemical separations started a few days later in a 
well-equipped nuclear laboratory in Harwell, Great Britain. They carried out a standard chemical separation of a mercury-fraction $(\mathrm{Hg})$, presumably carrying element $Z=112$ which belongs to the heavy end of the same group in the periodic table as $\mathrm{Hg}$ and which is also called eka-Hg. They produced a thin sample to study spontaneous fission events using a well-accepted detection-method for the observation of these fission events, called "Spark-Jump" technique. In this sample they observed during the following 37 days 93 spontaneous fission events. Such an event rate is far beyond any reasonable background effect for an experienced „fission events counting” research group. The team also isolated chemical fractions from the Pt-, Au-, Tl-, and Pb-tracers in the sample, in order to look for the respective eka-elements $Z=110,111,113$, and 114. They did not report finding any trace of a spontaneous fission activity in any of these other samples. Spontaneous fission is observed only for heavy elements starting with thorium $(Z=90)$ and beyond, so the selective focus on eka-elements having $\mathrm{Z} \geq 110$ is clear.

This result was discussed world-wide; however, the international science community was not convinced. During the following decades Marinov and his co-workers published a long series of papers on this subject. The international science community, however, remained unconvinced, as shown in [1]. The central reasons for the rejection of Marinov's claim for the discovery of element 112 have been: The original experiment was never reproduced in exactly the same way as it was done in 1971, and it was not expected that one could find heavy elements in irradiations of heavy targets with protons.

One should remember: In those days around the year 1971, the discovery of new elements was essentially the domain of HEAVY ION accelerators in a few laboratories around the world. The heaviest nuclides known were short-lived and from elements up to $Z=105$. The exciting and complex history of this research up to the year 1985 has been systematically described in a review by Seaborg and Loveland in [4]. It was generally assumed that no new heavy element was supposed to be produced by proton induced reactions. The simultaneous search for long-lived Superheavy Elements in NATURE up to the year 1985 had been systematically described in a review by Flerov and Ter-Akopyan in [5].

Another reason for the rejection of the Marinov approach had technical as well as logistic aspects: The highly radioactive target had to be transported from CERN in Switzerland to another laboratory in Europe within a short time of less than about 1 week. This was arranged once and could not be repeated. The reasons are not scientific, but rather of logistic origin and due to other real obstacles, such as radiation protection issues. In short: it was not possible. These logistic and security aspects, as well as the hostility towards the entire Marinov-approach made an exact reproduction of the original experiments reported in [3] impossible to date.

\subsection{Problem 2}

Ref. [1] (re: Marinov) quoting page 1337:

"In his later (second) challenge, Brandt [[25]] alludes to two lines of evidence 
for unusually high cross-sections of secondary particles. Even if the JWP accepted his lines of evidence-which it does not-this would not bring the original claim for discovery of the element with atomic number 112 by the Marinov group any closer to satisfying the criteria for discovery".

Problem 2: Reference [[25]] is cited as having only one author, whereas the paper really has 16 authors. The authors of [[25]] never "alluded" to anything but rather they described observed experimental facts. These facts are due to "Unresolved Problems" as published by these same 16 authors in [6].

Fortunately the findings of [[25]] have been properly described and accepted in an official letter of Professor P. J. Karol (the corresponding author of [1]), dated February 13, 2012, to Professor H. Stöcker (GSI Helmholtzzentrum für Schwerionenforschung GmbH, 64291 Darmstadt, Germany). The essential sentences in this letter are (quote): Secondly, comment on the studies in our publication was judgmental, but the criticism was directed solely at the interpretation of the experimental results and their pertinence to heavy element discovery claims made by A. Marinov and collaborators. There was no intent to disparage, in any way, the experimental results themselves. Indeed, I have been closely following Reinhard Brandt's work in this area for years, catalyzed by the early publication "Enhanced production of ${ }^{24} \mathrm{Na}$ by wide-angle secondaries produced in the interaction of relativistic carbon ions with copper, Phys. Rev. C45, 1194 (1992), by R. Brandt, G. Dersch, E.M. Friedlander, G. Haase, M. Heck V.S. Butsev, M.I. Krivopustov, B.A. Kulakov, E.-J. Langrock, F. Pille, H.H. Cui, and E. Ganssauge". (End of quote)

These facts, called "Unresolved Problems" in [6], have been studied further during recent years and published in [7]-[12]. The emphasis of this research has shifted over the years from "Na-production in copper by relativistic ions" to the measurement of excess neutron production which is far beyond theoretical model calculations in THICK $\mathrm{Cu}$ targets (or heavier elements such as e.g. $\mathrm{Pb}, \mathrm{U}$ ) irradiated with high-energy ions. The least understood experimental finding always was: Observed neutron fluxes above a certain projectile energy are about a factor of two LARGER than the fluxes calculated by any theoretical model. Consequently, the problems mentioned as being UNRESOLVED in [6] remain UNRESOLVED until today. The present state of our investigations is presented in [11] and [12]. These continued studies employed radiochemical experimental techniques together with studies using nuclear emulsion and correlated theoretical model calculations. Whereas radiochemical measurement of reaction products yields a very precise integral picture of all reaction products, nuclear emulsion shows differential pictures of single projectile-target interactions. With emulsion one can determine nuclear charges, energies and multiplicities of particles in the exit channel and follow the dynamics of several generations of products from one primary interaction. Using this combination of different research techniques, various irradiations at high-energy heavy ion accelerators were carried out in several laboratories. The combination of research opportunities leads to our present conclusions about unresolved problems in high-energy 
irradiations of thick targets:

- using radiochemical research tools one observes too many neutrons being produced; an enhancement factor of 2 to 3 is observed, as compared to various model calculations.

- in nuclear emulsion irradiated with high-energy heavy ions one observes a large fraction of BURST-interactions along with the expected spallation interactions. These BURSTS appear to have too few tracks from low-energy particles $(\mathrm{E}<0.5 \mathrm{GeV})$ and too many high-energy tracks $(\mathrm{E}>0.5 \mathrm{GeV})$. The detailed description of these experiments can be found in [11] and [12].

No complete understanding can be presented for those experimental observations which are described as "unresolved problems" in [6]. Nevertheless, these studies may become relevant for possible future experiments using heavy ion accelerators, including those presently under construction. According to our studies it is clearly predictable that in future experiments BURST-interactions with excess neutron production will occur, for example inside a large uranium target. It appears that no theoretical model can properly simulate that situation. The authors know of only one experiment, where massive uranium targets have been irradiated with heavy ions beams, like $44 \mathrm{GeV}{ }^{12} \mathrm{C}$. Ref. [7] describes this experiment and shows that the irradiation produced a secondary neutron flux, about a factor of two larger than predicted by any model calculation.

\subsection{Problem 3}

Ref. [1] quoting page 1337:

"The Marinov collaboration also recently claimed discovery [[24] [26] [27]] of several long-lived, naturally occurring, very neutron-deficient thorium isomeric states, e.g., ${ }^{210}$ Th and long-lived isotopes (allegedly Rg) with mass numbers 261 and 265 in natural Au at the sub-ppb level of abundance. These claims are based on mass spectroscopic data where an inductively coupled plasma ion source is used and very low background count rates are observed".

Problem 3: Ref. [[24]] reads (quote): "A. Marinov, I. Rodushkin, A. Pape, Y.Kashiv, D. Kolb. R. Brandt, R. Gentry, H.W. Miller, L. Halicz, I.Segal. (unpublished, submitted to Phys. Rev. Lett. 2007)". This paper never appeared in Phys. Rev. Lett. and it is not available for reference.

Note: Ref. [[26]] introduces a rather original application by Marinov et al. as new research tool-an "inductively coupled plasma-sector field mass spectrometer (ICP-SFMS)"-into the search for very heavy low-intensity nuclear species in nature. This type of research tool requires a very long half-life for these nuclides of much more than $10^{7}$ years in order to be observable. The description of the details is accurate for the study of a thorium target. Ref. [[26]] is listed as [13] in this paper.

\section{Outlook}

We wish to submit one suggestion to the "IUPAP/IUPAC Joint Working Group 
JWG)" mentioned in the Introduction: The scientific state-of-art in analytical (chemical and physical) investigations and in theoretical methods used for these experiments has changed considerably during the last twenty-five years (see [1]). The ICP-SFMS technique is just one of the new modern analytical tools. One should consider that presently unexplained experimental findings may indicate novel and unexpected reaction paths leading to unexpected results.

Last but not least, one should remember that around 1970 not only Marinov et al. claimed the observation of $\mathrm{Z}=112$ in a European laboratory, but also Hoffmann et al. [14] unexpectedly claimed in America to have observed the isotope ${ }^{244} \mathrm{Pu}\left(\mathrm{T}_{1 / 2}=8 \times 10^{7}\right.$ a) in a terrestrial sample. Both observations have neither been reproduced, nor have they been accepted by the international science community.

\section{References}

[1] Barber, R.C., Gäggeler, H.W., Karol, P.J., Nakahara, H., Verdaci, E. and Vogt, E. (2009) Discovery of the Element with Atomic Number 112 (IUPAC Technical Report). Pure and Applied Chemistry, 81, 1331-1343. https://doi.org/10.1351/PAC-REP-08-03-05

[2] The IUPAC (International Union of Pure and Applied Chemistry issued on June 1, 2017 a Project (2017-014-2-200), Described in: https://iupac.org/projects/project-details/?project_nr $=2017-014-2-200$

[3] (3a) Marinov, A., Batty, C.J., Kilvington, A.I., Newton, G.W.A., Robinson, V.J. and Hemingway, J.D. (1971) Evidence for the Possible Existence of a Superheavy Element with Atomic Number 112. Nature (London), 229, 464-467.

(3b) Marinov, A., Batty, C.J., Kilvington, A.J., Weil, J.L., Friedmann, A.M., Newton, G.W.A., Robinson, V.J., Hemingway, J.D. and Mather, D.S. (1971) Spontaneous Fission Previously Observed in a Mercury Source. Nature (London), 234, 212-215. https://doi.org/10.1038/234212b0

[4] Seaborg, G.T. and Loveland, W.D. (1985) Transuranium Elements. In: Bromley, D.A., Ed., Treatise on Heavy Ion Science, Vol. 4, Plenum Press, New York, 255-329. https://doi.org/10.1021/ed062p463

[5] Flerov, G.N. and Ter-Akopyan, G.M. (1985) Superheavy Elements. In: Bromley, D.A., Ed., Treatise on Heavy Ion Science, Vol. 4, Plenum Press, New York, 331-399. https://doi.org/10.1007/978-1-4615-8097-3_3

[6] Brandt, R., Ditlov, V.A., Dwivedi, K.K., Ensinger, W., Ganssauge, E., Guo, S.-L., Haiduc, M., Hashemi-Nezhad, S.R., Khan, H.A., Krivopustov, M.I., Odoj, R., Pozharova, E.A., Smirnitzki, V.A., Sosnin, A.N., Westmeier, W. and Zamani-Valasiadou, M. (2008) Interactions of Relativistic Heavy Ions in Thick Heavy-Element Targets and Some Unresolved Problems. Physics of Particles and Nuclei, 39, 259-285. https://doi.org/10.1134/S1063779608020044

[7] Hashemi-Nezhad, S.R., Zamani-Valasiadou, M., Krivopustov, M.I., Brandt, R., Ensinger, W., Odoj, R. and Westmeier, W. (2011) Neutron Production in Thick Targets Irradiated with High-Energy Ions (Review Article). Physics Research International, 2011, Article ID: 128429. https://doi.org/10.1155/2011/128429

[8] Westmeier, W., Brandt, R., Hashemi-Nezhad, R., Odoj, R., Ensinger, W., Zamani-Valasiadou, M. and Sosnin, A. (2012) Correlations in Nuclear Interactions between $E_{C M} / u$ and Unexplained Experimental Observables. World Journal of Nuclear Science and Technology, 2, 125-132. https://doi.org/10.4236/wjnst.2012.24018 
[9] Ganssauge, E., Westmeier, W. and Brandt, R. (2013) Potential Correlations between Unexplained Experimental Observables and Hot Projectile-Like Fragments in Primary Interactions above $\mathrm{E}_{\mathrm{CM}} / \mathrm{u} \approx 150 \mathrm{MeV}$. World Journal of Nuclear Science and Technology, 3, 155-161. https://doi.org/10.4236/wjnst.2013.34026

[10] Brandt, R., Ditlov, V., Haiduc, M., Firu, E., Neagu, A., Ganssauge, E., Hashemi-Nezhad, R. and Westmeier, W. (2015) Two Ways of High-Energy Heavy-Ion Interactions: Spallation and Burst. World Journal of Nuclear Science and Technology, 5, 73-87. https://doi.org/10.4236/wjnst.2015.52007

[11] Hashemi-Nezhad, R., Brandt, R., Ditlov, V., Haiduc, M., Firu, E., Neagu, A.T., Ganssauge, E. and Westmeier, W. (2017) Further Studies of BURST and Spallation in High-Energy Heavy Ion Interactions. World Journal of Nuclear Science and Technology, 7, 35-57. https://doi.org/10.4236/wjnst.2017.71004

[12] Hashemi-Nezhad, S.R., Brandt, R., Ditlov, V., Firu, E., Ganssauge, E., Haiduc, M., Neagu, E.A. and Westmeier, W. (2017) Monte Carlo Studies of the Interaction of Relativistic Ions with Nuclear Emulsion. Nuclear Instruments and Methods in Physics Research, A841, 72-86.

[13] Marinov, A., Rodushkin, I., Kashiv, Y., Halicz, L., Segal, I., Pape, A., Gentry, R.V., Miller, H.W., Kolb, D. and Brandt, R. (2007) Existence of Long-Lived Isomeric States in Natural-Occurring Neutron Deficient Th Isotopes. Physical Review, C76, Article ID: 021303. https://doi.org/10.1103/PhysRevC.76.021303

[14] Hoffmann, D.C., Lawrence, F.O., Mewherter, J.L. and Rourke, F.M. (1971) Detection of Plutonium-244 in Nature. Nature (London), 234, 132-134.

https://doi.org/10.1038/234132a0 